Asian J. Med. Biol. Res. 2016, 2 (2), 213-220; doi: 10.3329/ajmbr.v2i2.29063

\author{
Asian Journal of \\ Medical and Biological Research \\ ISSN 2411-4472 (Print) 2412-5571 (Online) \\ www.ebupress.com/journal/ajmbr
}

Article

\title{
Distribution of sul genes and their variants in uropathogenic Escherichia coli isolated from two hospitals of Sabah
}

\author{
Zaw $\operatorname{Lin}^{1}{ }^{*}$, Yun Mei Lai ${ }^{1}$, Myo Thura Zaw ${ }^{1}$ and Ahmad Toha Samsudin ${ }^{2}$ \\ ${ }^{1}$ Department of Pathobiological and Medical Diagnostics, Faculty of Medicine and Health Science, Universiti \\ Malaysia Sabah \\ ${ }^{2}$ Department of Pathology, Hospital Queen Elizabeth, Kota Kinabalu, Sabah
}

*Corresponding author: Pathobiological and Medical Diagnostics Department, Faculty of Medicine and Health Sciences, Universiti Malaysia Sabah, Jalan UMS, Kota Kinabalu, 88400, Sabah, Malaysia. Phone: 320000 Ext: 611428; E-mail: zawlin@ums.edu.my

Received: 31 May 2016/Accepted: 16 June 2016/ Published: 30 June 2016

\begin{abstract}
Sulphonamides resistant strains are highly prevalent in uropathogenic Escherichia coli (UPEC) isolates. Sul genes encode sulphonamide resistance and are present on transferrable plasmids. Integrons (IGNs) are genetic elements containing integrase gene, attl site and gene cassettes which carry multiple antibiotic resistant genes. Class 1 integrons have been extensively studied because these were most prevalent among clinical isolates. In this study, UPEC isolates were determined for the antibiotic susceptibility patterns to four antibiotics commonly used for urinary tract infections, which include co-trimethoxazole (TMP-STX). Distribution of sul genes and integrase1 gene (intIl) was studied in TMP-STX resistant UPEC isolates by using multiplex polymerase chain reaction (mPCR). Sul genes variants were investigated by DNA sequencing of the whole open reading frame of sull and sul2 genes and PCR product of sul3 gene. Sul1, sul2 and sul3 genes were prevalent in $37(24.7 \%)$ of 150 UPEC isolates. IntIl is positive in 22 sul genes positive isolates. Of six isolates positive with sul2 genes, sul2(a) and sul2(b) variants, which were described in the previous study, in the four isolates and the two isolates respectively were observed. This is the first mPCR which investigates the prevalence of three sul genes and intIl in the UPEC clinical isolates from two hospitals of Sabah.
\end{abstract}

Keywords: uropathogenic Escherichia coli; sul genes; integrase 1 gene; sul2 variants; multiplex polymerase chain reaction; hospitals of Sabah

\section{Introduction}

The major problem in treatment of UTI is the resistance of UPEC to antimicrobial agents. The previous studies indicated that there is increasing trend of UPEC resistance to TMP-STX. In Europe, TMP-STX resistance rates among uropathogens increased from 0-5\% before 1990 to 9-26\% in 1999 and 2000 (Blahna et al., 2006).

After their clinical introduction in 1935, sulfonamides were used to treat bacterial and protozoal infections. Sulfonamides have generally been used in combination with diaminopyrimidines to overcome the rapid emergence of resistance since 1970s (Huovinen et al., 1995; Huovinen, 2001). The combination trimethoprimsulfamethoxazole is still commonly used in clinical practice and one of the drugs of choice for the treatment of urinary tract infections.

Dihydropteroate synthase (DHPS) is an enzyme important in the folic acid biosynthesis pathway. Competition of sulfonamides with the structural analog p-aminobenzoic acid for binding to dihydropteroate synthase (DHPS), results in inhibition of the formation of dihydrofolic acid (Sko"ld, 2000). Resistance to sulfonamides in E. coli can result from mutations in the chromosomal DHPS gene $(f o l P)$ as well as the acquisition of an alternative DHPS gene $(s u l)$. The protein encoded by sul gene has a lower affinity for sulphonamides 
(SwedbergandSko"ld, 1980; Swedberg et al., 1993; Ra ${ }^{\circ}$ dstro"m and Swedberg, 1998; Vedantam et al., 1998; Sundstro"m et al., 1998).

Three alternative sulfonamide resistance DHPS genes (sul1, sul2 and sul3) in gram-negative bacteria have been described. The DNA sequences of sull and sul2 from E. coli are different from all the known chromosomal DHPS genes from E. coli and other bacteria and their origin remains unknown (Ra ${ }^{\circ} \mathrm{dstro} " \mathrm{~m}$ and Swedberg, 1998). Sull has been found on large conjugative plasmids carrying on class 1 integrons ( $\mathrm{Ra}^{\circ} \mathrm{dstro}$ “ $\mathrm{m}$ et al., 1991; Antunes et al., 2005; Hammerum et al., 2006; Trobos et al., 2008). The sul2 gene has also been recently found on a wide range of large conjugative plasmids as well as small non-conjugative plasmids (Hammerum et al., 2006; Trobos et al., 2008; Bean et al., 2009).

Perreten and Boerlin described a new alternative sulfonamide resistance determinant called sul3 which is probably acquired by $E$. coli from a distantly related organism. The presence of sul3 on different plasmids in different $E$. coli clones, this gene was flanked by two insertional elements and a distinct DNA sequence of it suggested that it was a new sul gene (Perreten and Boerlin, 2003)

Rapid transmission of drug resistance in bacterial pathogens is the consequence of the widespread use of antibiotics as well as transfer of drug resistance determinants mediated by plasmids, transposons and gene cassettes in IGNs. The presence of variable no. of drug resistance genes in their gene cassettes makes the genetic organizations of IGNs diverse (Li et al., 2013). An IGN is defined as a genetic element that consists of three parts. The parts are the gene which encodes integrase that mediates site-specific recombination events, the site, attI, at which gene cassettes can be integrated by site-specific recombination and gene cassettes. Although IGNs themselves are not mobile, the integrase enzyme excises and integrates the gene cassettes from and into the IGN (Bennett, 1999; Fluit and Schmitz, 2004).

There are five classes of IGN and the first three classes are resistant integrons while class 4 and 5 are superintegrons. The most prevalent IGN is class I and it is present in gram-negative bacteria including E. coli. Class 1 IGNs have been extensively studied because these were most prevalent among clinical isolates as well as commensals out of three classes (Bass et al., 1999; Chang et al., 2000; Mazel et al., 2000; Naas et al., 2000; Sundeand Sorum, 2000)

Out of three sul genes, the sul2 gene has been reported to be the most prevalent gene in Escherichia coli. This gene was highly conserved, and is usually carried on large conjugative resistance plasmids. Sul2 genetic variation in E.coli isolates from animal, meat and human clinical samples and normal human was previously studied. Six point mutations were observed with four synonymous mutations and two non-synonymous mutations (Trobos et al., 2009). However, there were no reports of sull and sul3 genetic variants.

In this study, UPEC isolates were determined for the antibiotic susceptibility patterns to four antibiotics. Multiplex PCR of three sul genes and intIl gene was set up to screen TMP-STX resistant UPEC and application of this PCR method to detect three sul genes in UPEC isolates from two hospitals, Hospital Queen Elizabeth and Hospital Papar, of Sabah State, Malaysia. Most of the sul genes were present on IGN and detection of class 1 integrase was included in this study. In addition, DNA sequencing of three sul genes were undertaken to find out the variants of sul genes.

\section{Materials and Methods \\ 2.1. Samples and controls}

One hundred and fifty UPEC isolates stocked in Microbiology Laboratory, Faculty of Medicine and Health Sciences, Universiti Malaysia Sabah were investigated for antibiotic susceptibility tests in this study. These UPEC isolates were derived from urine samples sent to microbiology laboratory (for culture and sensitivity) of two hospitals, Hospital Queen Elizabeth and Hospital Papar which are located around Kota Kinabalu, Sabah and we have checked for significant bacteriuria to include in the criteria for UPEC. The study was done on the urine samples collected between January and June, 2013.

Positive controls for sull, sul2, sul3, intIl were pKTN117, pKTN118 and pKTN 119, pKTN 120 respectively. These plasmids were constructed with the PCR products of these genes, which were cloned into TopoTA plasmids. The genes inside the plasmids were sequenced, aligned with the sequences downloaded from the NCBI website and the DNA sequences were observed to be homologous. Escherichia coli ATCC 25922 was used as the negative control in this study.

\subsection{Disc diffusion method}

The stocked UPEC isolates were cultured on MacConkey agar and disc diffusion method was performed on Mueller-Hinton agar plates, according to Clinical and Laboratory Standards Institute (CLSI) guidelines to 
perform antibiotic susceptibility test (AST) (CLSI, 2012).The antibiotic discs used in this study were cotrimethoxazole (TMP-STX), ciprofloxacin (CIP), gentamycin (GM) andcefotaxime (CTX).

\subsection{Determination of minimum inhibitory concentration}

This procedure was performed using the agar dilution method as described by the Clinical and Laboratory Standards Institute (CLSI) guidelines (CLSI, 2012). The minimal inhibitory concentration (MIC) is defined as the lowest antimicrobial concentration able to totally inhibit bacterial growth. E. coli ATCC 25922 was used as a negative control for both disc diffusion method and MIC study (CLSI, 2012).

\subsection{Multiplex PCR for detection of sull, sul2, sul3 and intII genes}

Those isolates having resistance to TMP-STX were studied for detection of sull, sul2, sul3 genes and intllby multiplex PCR. The bacterial isolates were inoculated in $3 \mathrm{ml}$ of Luria-Bertani broth and incubated at $37^{\circ} \mathrm{C}$ for 18 hrs. The bacterial DNA was extracted by boiling method (Abdallah et al., 2011; Ifeanyi et al., 2015).

The primer sets for sul1, sul2, sul3 genes, intIl gene and 16srRNA gene were mentioned in Table 1 (Kerrn et al., 2002; Gundogdu et al., 2011). The 16s rRNA gene was amplified in each reaction as an internal positive control for confirmation of E. coli. The ingredients for multiplex PCR were $6 \mu \mathrm{L}$ of DNA template, 1x PCR buffer, $3 \mathrm{mM} \mathrm{MgCl} 2,0.4 \mathrm{mM}$ dNTPs, $1.5 \mathrm{U}$ of Taq polymerase, $0.4 \mathrm{mM}$ each of $16 \mathrm{srRNA}$ gene, sull, sul 2 and intII sets of primers, $0.8 \mathrm{mM}$ of sul 3 forward and reverse primers and sterile distilled water was added to make total $25 \mu \mathrm{l}$ of PCR mixture. The PCR conditions were $94^{\circ} \mathrm{Cx} 5 \mathrm{~min}, 30$ cycles of $94^{\circ} \mathrm{C} \mathrm{x} 30 \mathrm{~s}, 58^{\circ} \mathrm{C} \mathrm{x} 30 \mathrm{~s}, 72^{\circ} \mathrm{C} \mathrm{x}$ $1 \mathrm{~min}$ and a final extension of $72^{\circ} \mathrm{C} \times 7 \mathrm{~min}$.

Amplification was performed using Applied Biosystems Thermocycler and First base Taq DNA polymerase. The sizes of PCR amplicons were checked by $1.5 \%$ TAE agarose gel electrophoresis and Alpha Imager ${ }^{\circledR}$ HP System gel documentation apparatus after the gel was stained with ethidium bromide.

\subsection{PCR for sull and sul2 whole gene sequence for detection of sul gene variants}

For sull gene and sul2 gene the whole sequence amplification, the primer setssullws and sul2ws primer sets shown in Table 1 were used. The primers sets were taken from NCBI website taking care of the precautions in primer design. The PCR ingredients were $6 \mu \mathrm{L}$ of DNA template, 1x PCR buffer, $3 \mathrm{mM} \mathrm{MgCl}_{2}, 0.4 \mathrm{mM} \mathrm{dNTPs}$, $1.5 \mathrm{U}$ of Taq polymerase, $0.4 \mathrm{mM}$ of each forward and reverse primers of sullws for sull. The PCR conditions were $94^{\circ} \mathrm{C}$ x $5 \mathrm{~min}, 30$ cycles of $94^{\circ} \mathrm{C}$ x $30 \mathrm{~s}, 56^{\circ} \mathrm{C}$ x $30 \mathrm{~s}, 72^{\circ} \mathrm{C}$ x $1 \mathrm{~min}$ and a final extension of $72^{\circ} \mathrm{C}$ x $5 \mathrm{~min}$. The PCR ingredients were the same for sul 2 gene with the exception that forward and reverse primers of sul2ws were used. The PCR conditions were $94^{\circ} \mathrm{C}$ x $5 \mathrm{~min}, 35$ cycles of $94^{\circ} \mathrm{C}$ x $45 \mathrm{~s}, 54^{\circ} \mathrm{C} \mathrm{x} 45 \mathrm{~s}, 72^{\circ} \mathrm{C}$ x $1 \mathrm{~min}$ and a final extension of $72^{\circ} \mathrm{C}$ x $5 \mathrm{~min}$. Amplification and gel documentation were the same as mPCR method. For the sul3 gene DNA sequence, PCR products of MPCR method were used.

\subsection{DNA sequencing of PCR products}

DNA sequencing of PCR products was performed with Applied Biosystems highest capacity-based genetic analyzer using Big Dye ${ }^{\circledR}$ Terminator v3.1 cycle sequencing kit.

\subsection{Alignment of DNA sequences to find single nucleotide polymorphisms (SNPs)}

The resulting DNA sequences were analyzed with A plasmid Editor (ApE) software and nucleotide sequences were aligned with wild type sul gene sequences downloaded from NCBI website to detect sul genes variants and nucleotide sequence numbers were counted according to wild type sequences.

\section{Results}

\subsection{Results of AST and MIC value}

One hundred and fifty UPEC isolates were tested for antibiotic susceptibility patterns with four antibiotics commonly used for UTI, which were mentioned in the method. Out of 150 isolates, forty, five, four and three isolates were resistant to TMP-STX, gentamycin, ciprofloxacin and cefotaxime, respectively. In case of MIC determination for the TMP-STX resistant isolates, it was observed to be $128 / 2432 \mu \mathrm{g} / \mathrm{mL}$

\subsection{Results of mPCR setting up and screening of sul genes and intII gene in TMP-STX resistant UPEC isolates \\ After setting up of multiplex PCR (Figure 1), all the TMP-STX resistant isolates were investigated for sul genes and intIl gene. The distribution of sul genes were mentioned in Table 2. Presence of at least one of the sul genes was observed in 37 (92.5\%) of 40TMP-STX resistant isolates. Sul2 gene was most prevalent (68\%), sull gene}


was $52 \%$ and sul3 gene was the least (16\%). Of total 22 isolates positive for intIl, 11 were sull positive, 13 were sul2 positive and 5 were sul3 positive (Table 2).

There was absence of isolate having sull, sul2 and sul3 genes together. An interesting finding was no coexistence of sull and sul3 in the studied UPEC isolates.

Table 1. Primer sequences for sul genes, intI1 gene, 16srRNA gene for E. coli, sul1ws and sul2ws.

\begin{tabular}{|c|c|c|}
\hline Target gene & Sequence of Primers & Expected size of PCR products \\
\hline Sull & F: 5'-CTTCGAT GAGAGCCGGCGGC-3' & 433 bp \\
\hline & R: 5'-GCAA GGCGGAAACCCGCGCC-3' & (Kerrn et al., 2002) \\
\hline Sul2 & F: 5'- GCGCTCAAGGCAGATGGCATT-3' & 293 bp \\
\hline & R: 5'-GCGT TTGATACCGGCACCCGT-3' & (Kerrn et al., 2002) \\
\hline Sul3 & F: 5'-GAGCAAGATTTTTGGAATCG-3' & 790 bp \\
\hline & R:5'- CATCTGCAGCTAACCTAGGGCTTTGGA-3' & (Gundogdu et al., 2011) \\
\hline IntII & $\begin{array}{l}\text { F: 5'-CAGTGGACATAAGCCTGTTC-3' } \\
\text { R: 5'-CCCGAGGCATAGACTGTA-3' }\end{array}$ & $\begin{array}{l}160 \mathrm{bp} \\
\text { (Gundogdu et al. 2011) }\end{array}$ \\
\hline 16S rRNA (E.coli) & $\begin{array}{l}\text { F: 5'-GCGGACGGGTGAGTAATGT-3', } \\
\text { R: 5'-TCATCCTCTCAGACCAGCTA-3, }\end{array}$ & $200 \mathrm{bp}$ \\
\hline Sullws & $\begin{array}{l}\text { F: 5'-ATGGTGACGGTGTTCGGCA-3' } \\
\text { R: 5'-CTAGGCATGATCTAACCCTC-3' }\end{array}$ & $840 \mathrm{bp}$ \\
\hline Sul2ws & $\begin{array}{l}\text { F: 5'-ATGAATAAATCGCTCATCA-3' } \\
\text { R: 5'-TTAACGAATTCTTGCGGT-3' }\end{array}$ & $\begin{array}{l}816 \text { bp } \\
\text { (this study) }\end{array}$ \\
\hline
\end{tabular}

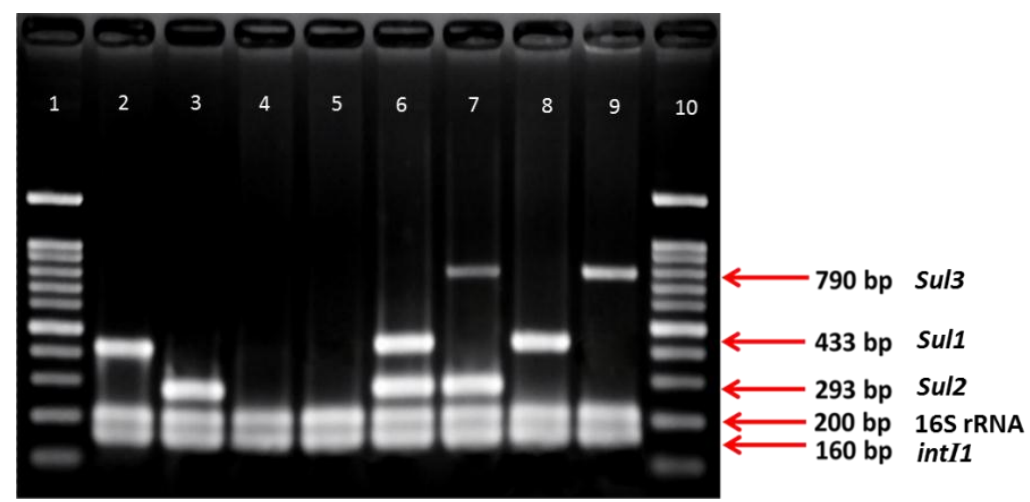

Figure.1. Gel electrophoresis picture of multiplex PCR for three sul genes, intl lgene and 16s rRNA of $E$. coli. All the sample lanes are 16s rRNA and intI1 gene positive. Lane 2,8 aresull positive isolate. Lane 3 is sul2 positive isolate. Lane 4 and 5 have no sul genes. Lane 6 is sull and sul2 positive isolate. Lane 7 is sul2 andsul3 positive isolate. Lane 9 is sul3 positive isolate. Lane $1 \& 10$ are100bp ladder molecular marker. The sizes of PCR products are 433bp for sull gene, 293bp for sul2 gene and 790bp for sul3 gene while $200 \mathrm{bp}$ for 16s rRNA gene and 160bp for intI1 gene.

Table 2. Distribution of sul genes in TMP-STX resistant isolates and intI1 positive isolates which were TMP-STX resistant.

\begin{tabular}{lcccccccccc}
\hline $\begin{array}{l}\text { Types } \\
\text { Isolates }\end{array}$ & of & sul1 & sul2 & sul3 & $\begin{array}{l}\text { sul1\& } \\
\text { sul2 }\end{array}$ & $\begin{array}{l}\text { sul1\& } \\
\text { sul3 }\end{array}$ & $\begin{array}{l}\text { sul2\& } \\
\text { sul3 }\end{array}$ & $\begin{array}{l}\text { sul1, } \\
\text { sul2 \& } \\
\text { sul3 }\end{array}$ & $\begin{array}{l}\text { Total no. of } \\
\text { sul genes }\end{array}$ & $\begin{array}{l}\text { Total } \\
\text { no. } \\
\text { isolates } \\
\text { of }\end{array}$ \\
\hline $\begin{array}{l}\text { UPEC } \\
\text { intII }\end{array}$ & & 8 & 12 & 4 & 11 & 0 & 2 & 0 & $37(* 22.8 \%)$ & 162 \\
isolates & Positive & 5 & 5 & 3 & 7 & 0 & 2 & 0 & 22 & - \\
\hline
\end{tabular}

Footnote - *Percentage of sul genes in total UPEC isolates 


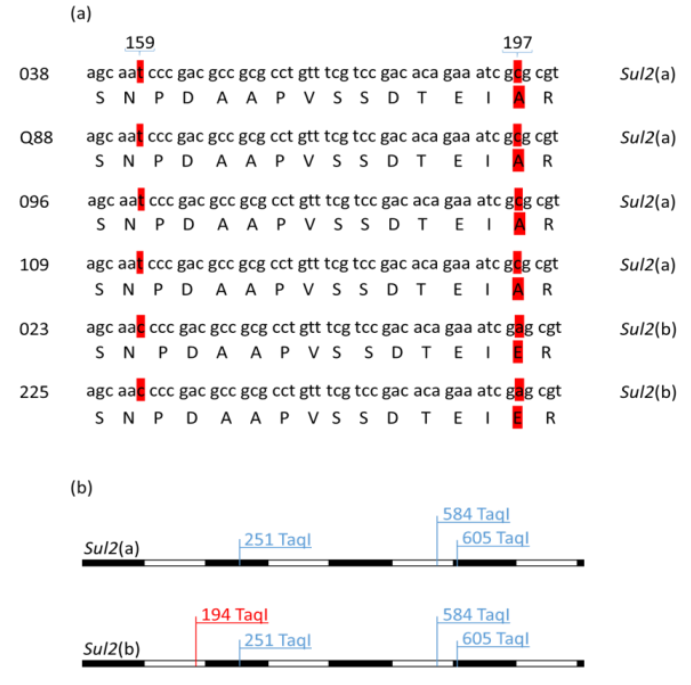

Figure 2. Six UPEC isolates with two sul2 variants and differentiation of these variants by PCR-RFLP analysis (a) Nucleotide sequence alignment among six isolates with two sul2 variants, sul2(a) and sul2(b)is shown. Isolates no. 038,Q88,096,109 have sul2(a) variants and isolates no. 023 and 225 havesul2(b) variants with change of amino acid from $A$ to $E$ (non-synonymous nucleotide change at $197 \mathrm{NP}$ ) (b) Restriction map of sul2(a) variant and sul2(b) variant are shown for PCR-RFLP analysis by TaqI restriction enzyme to differentiate sul2 variants. Sul2(b) variant has extra-TaqI restriction site.

\subsection{Investigation of sul genes variants}

Altogether, six of each sul genes were undertaken for DNA sequence analysis and all the sull and sul3 genes were totally identical to the sequence of NCBI downloaded respective sequences. However, two isolates positive with sul2 genes have one synonymous mutation at nucleotide position (NP) no.159 ( $\mathrm{T}$ to $\mathrm{C}$ variant) and another non-synonymous mutation at NP no.197 (C to A variant), which changed from amino acid alanine to glutamic acid. Trobos et al. (2009) have named sul2(a) for T at NP 159 and C at NP 197 (TC variant) and sul2(b) for C at NP 159 and A at NP 197 (CA variant), which were observed in their study. As we followed the system described in that study, sul2(a) and sul2(b) variants in the four isolates and the two isolates respectively were observed among six sul2 genes positive isolates sequenced (Figure 2 (a)). Mutation at nucleotide sequence no. 197 could be investigated by PCR-RFLP analysis with Taq I restriction enzyme giving rise to 194 bp DNA fragment in case of sul2(b) variant gene while $251 \mathrm{bp}$ DNA fragment was observed in sul2(a) variant (data not shown) (Figure 2 (b)).

\section{Discussion}

This is the first study to use multiplex PCR for detection of three sul genes in human UPEC isolates obtained from hospitals of Sabah. There has been a study on three sul genes in E. coli isolates from healthy humans, pork and pigs in Denmark and clinical samples were not included and the study did not mention that multiplex PCR was used. However, the literature has shown one PCR method for the four genes sull, sul2, sul3 and intIl indicating that their PCR was multiplex one (Hammerum et al., 2006).

In addition, Gundogdu et al. (2011) studied all three sul genes in UPEC. However, in their PCR sull and sul2 were detected in one PCR reaction while sul 3 was detected in another PCR reaction.

Wu et al. (2010) studied on 109 TMP-STX resistant E. coli strains isolated from pig feces, pig carcasses and human stools. In their study, 26 isolates from human stool were resistant to TMP-STX. Of these isolates, sul2 gene was the most prevalent gene followed by sull gene. There was no coexistence of sull and sul3 in these human isolates. Sull and sul2 coexistence was higher while sul2 and sul3 genes were together in lesser no. of isolates. These above mentioned findings were consistent with the findings in our study. Teichmann et al. (2014) indicated that sul2 gene was highly prevalent in TMP-STX resistant UPEC isolates up to $67 \%$ in their study. In this study, sul2 genes were present in $68 \%$ of TMP-STX resistant isolates showing that sul2 genes was more prevalent than two other sul genes.

IGNs are capable of collecting of promoter-less gene cassettes through the actions of specialized site-specific recombination enzymes, intl. By mean of these mobile elements such as plasmids and transposons, IGNs can be horizontally transferred drug resistant genes between bacterial species commonly within the Enterobacteriaceae 
family (Hall et al., 2003). Therefore, in this study screening of intIl gene was undertaken together with sul genes in mPCR assay.

In the Danish study on E. coli isolates from UTI cases and bacteremia cases, sull, sul2 and intIl gene were included in the multiplex PCR and sul2 gene was the most common gene among these. The study did not include the sul3 gene. In that study, 97\% of TMP-STX resistant isolates had carried sul genes and $96 \%$ of sull gene positive strains had intIlgene (Kernn et al., 2002). In case of Class 1 integron, sull gene was commonly located at the 3' conserved region which is downstream of gene cassettes (Carattoli, 2001).In our study, 92.5\% of TMP-STX resistant isolates had positive for one of sul genes and 53\% of sull gene positive strains were positive for intIl gene.

Trobos et al. (2009) had studied on sul2 genetic variation in E. coli isolates from animal, meat and human clinical samples and normal human. Six point mutations were observed in 68 samples. The most common variant is sul2(a) and the second common variant was sul2(b) in their study. TC variants rarely contained an extra non-synonymous mutation at position 427 (A instead of G) and this mutation was named sul2(c) in that study and this variant was observed in two isolates. Other three synonymous mutations occurred at 288,489 and 672 nucleotide positions and each mutation was present in only one isolate.

Presence of the sul2 gene without much genetic variation in both human and animal isolates suggests horizontal transfer of the gene. Because only two to three point mutations, it did not indicate genetic drift and has been concluded by researchers that this had occurred within a short time (Sorum H and L'Abee-Lund, 2002). As an alternative hypothesis, it can be assumed that DHPS enzyme cannot tolerate many changes to its amino acid sequence (Trobos et al., 2009).

In this study, TMP-STX resistant UPEC were studied for distribution of sull, sul2 and sul3 genes and their variants because we have observed that sul genes positive isolates had consistent AST pattern and MIC values. These data support the information there is no much genetic variation of sul genes at the DNA level. However, the previous work in Denmark indicated that there were sul2 variants. Therefore it will be an interesting information if we find sul gene variants in our study.

Regarding two sul2 gene variants, the two variants observed in this study were included and the most common variants found in the other study (Trobos et al., 2009). The observed variants we observed in this study were common in human clinical isolates in their study and sul2(a) variant is more prevalent compared with variant sul2(b) in both studies. Although geographical regions were far away between Denmark and Sabah, Malaysia, it is surprising that the common two variants existing in sul2 gene in the two countries were consistent. We need further information on sul2 gene variants to draw a firm conclusion.

\section{Conclusions}

Up to date, there were no mPCR technique which can detect three sul genes and intll in isolates from UTI cases of hospitals in Malaysia. This study will be beneficial for the microbiologists to detect sulphonamide or TMPSTX resistant strains because PCR technique provides rapid and reliable results. UPEC isolates in this study were not highly resistant to gentamycin, ciprofloxacin and third generation cephalosporin indicating that antibiotics restriction policy was well controlled in the hospitals of Sabah. Furthermore, sul2(a) and sul2(b) variants could be investigated by PCR-RFLP analysis using Taq I restriction enzyme.

\section{Acknowledgements}

We would like to thank Professor Dr. Zainal Arifin Mustapha, Dean, Faculty of Medicine and Health Sciences, University Malaysia Sabah for the continuous support throughout the whole research project. This work is supported by Rural Medicine Research Unit Grant code no. 2102-RMRU-001.

\section{Conflict of interest}

None to declare.

\section{References}

Abdallah KS, Y Cao and DJ Wei, 2011. Epidemiologic Investigation of Extra-intestinal pathogenic E. coli (ExPEC) based on PCR phylogenetic group and fim $\mathrm{H}$ single nucleotide polymorphisms (SNPs) in China. Int. J. Mol. Epidemiol. Genet.,2: 339-353.

Antunes P, J Machado, JC Sousa and L Peixe, 2005. Dissemination of sulphonamide resistance genes (sull, sul2, and sul3) in Portuguese Salmonella enterica strains and relation with integrons. Antimicrob. Agents. Chemother., 49:836-839. 
Bass L, CA Liebert, MD Lee, AO Summers, DG White, SG Thayer and JJ Maurer, 1999. Incidence and characterization of integrons, genetic elements mediating multiple-drug resistance, in avian Escherichia coli. Antimicrob. Agents. Chemother., 43:2925-2929.

Bean DC, DM Livermore andLM Hall, 2009.Plasmids imparting sulphonamide resistance in Escherichia coli: implications for persistence. Antimicrob. Agents. Chemother., 53:1088-1093.

Bennett PM, 1999.Integrons and gene cassettes: a genetic construction kit for bacteria. J. Antimicrob. Chemother., 43:1-4.

Blahna MT, CA Zalewski, J Reuer, G Kahlmeter, B Foxman and CF Marr, 2006. The role of horizontal gene transfer in the spread of trimethoprim-sulfamethoxazole resistance among uropathogenic Escherichia coli in Europe and Canada. J. Antimicrob. Chemother., 57: 666-672.

Carattoli A, 2001. Importance of integrons in the diffusion of resistance. Vet. Res., 32:243-259.

Chang CY, LL Chang, YH Chang, TM Lee and SF Chang, 2000 Characterisation of drug resistance gene cassettes associated with class 1 integrons in clinical isolates of Escherichia coli from Taiwan, ROC.J. Med. Microbiol., 49: 1097-1102.

Clinical and Laboratory Standards Institute, 2012. Performance Standards for Antimicrobial Susceptibility Testing; Twenty-Second Informational Supplement. 32: M100-S22.

Fluit AC and FJ Schmitz, 2004.Resistance integrons and super-integrons. Clin. Microbiol. Infect., 10:272-288.

Gundogdu A, YB Long, TL Vollmerhausen and M Katouli, 2011.Antimicrobial resistance and distribution of sul genes and integron-associated intI genes among uropathogenic Escherichia coli in Queensland, Australia. J. Med. Microb., 60:1633-1642.

Hall MAL, HEM Blok, ART Donders, A Paauw, AC Fluit and J Verhoe, 2003. Multidrug resistance among Enterobacteriaceae is strongly associated with the presence of integrons and is independent of species or isolate origin. J. Infect. Dis., 187:251-259.

Hammerum AM, D Sandvang, SR Andersen, AM Seyfarth, LJ Porsbo, N Frimodt-Moller and OE Heuer, 2006. Detection of sull, sul2 and sul3 in sulphonamide resistant Escherichia coli isolates obtained from healthy humans, pork and pigs in Denmark. Int. J. Food. Microbiol., 106:235-237.

Huovinen P, 2001. Resistance to trimethoprim-sulfamethoxazole. Clin. Infect. Dis., 32:1608-1614.

Huovinen P, L Sundstro G Swedberg and O Sko"ld, 1995.Trimethoprim and sulfonamide resistance. Antimicrob.. Agents. Chemother.,39:279-289.

Ifeanyi CIC, NF Ikeneche, BE Bassey, N Al-Gallas, RB Aissa and A Boudabous, 2015. Diarrheagenic Escherichia coli pathotypes isolated from children with diarrhea in the Federal Capital Territory Abuja, Nigeria. J. Infect. Dev. Ctries., 9:165-174.

Kerrn MB, T Klemmensen, N Frimodt-Møller, and F Espersen, 2002. Susceptibility of Danish Escherichia coli strains isolated from urinary tract infections and bacteraemia, and distribution of sul genes conferring sulphonamide resistance. J. Antimicrob. Chemother., 50:513-516.

Li B, Y Hu, Q Wang, Y Yi, PCY Woo, H Jing, B Zhu and C H Liu, 2013. Structural Diversity of Class 1 Integrons and Their Associated Gene Cassettes in Klebsiella pneumoniae Isolates from a Hospital in China. PLOS ONE, 8:1-9.

Mazel D, B Dychinco, VA Webb and J Davies, 2000. Antibiotic resistance in the ECOR collection: integrons and identification of a novel aad gene. Antimicrob. Agents. Chemother., 44: 1568-1574.

Naas T, F Benaoudia, S Massuard and P Nordmann, 2000. Integron-located VEB-1 extended-spectrum betalactamase gene in a Proteus mirabilis clinical isolate from Vietnam. J. Antimicrob. Chemother., 46:703-711.

Perreten V and P Boerlin, 2003. A new sulfonamide resistance gene (sul3) in Escherichia coli is widespread in the pig population of Switzerland. Antimicrob. Agents. Chemother., 47:1169-1172.

$\mathrm{Ra}^{\circ}$ dstro“m P, G Swedberg and O Sko"ld, 1991. Genetic analyses of sulphonamide resistance and its dissemination in gram-negative bacteria illustrate new aspects of $\mathrm{R}$ plasmid evolution. Antimicrob. Agents. Chemother. 35:1840-1848.

Sko"ld O, 2000. Sulfonamide resistance: mechanisms and trends. Drug. Resist. Update, 3:155-160.

Sorum H and TM L'Abee-Lund, 2002. Antibiotic resistance in food related bacteria - a result of interfering with the global web of bacterial genetics. Int. J. Food.Microbiol.,78:43-56.

Sunde M and H Sorum, 2000. Characterization of integrons in Escherichia coli of the normal intestinal flora of swine. Microb. Drug. Resist., 5:279-287.

Sundstro"m L, P Ra ${ }^{\circ}$ dstro“m, G Swedberg and O Sko"ld, 1988. Site-specific recombination promotes linkage between trimethoprim- and sulphonamide resistance genes. Sequence characterization of $d h f r V$ and sulI and a recombination active locus of Tn21. Mol. Gen. Genet., 213:191-201. 
Swedberg G, C Ferme'r and O Sko"ld, 1993. Point mutations in the dihydropteroate synthase gene causing sulfonamide resistance. Adv. Exp. Med. Biol.,338:555-558.

Swedberg G and O Sko"ld, 1980. Characterization of different plasmid borne dihydropteroate synthases mediating bacterial resistance to sulfonamides. J. Bacteriol. 142:1-7.

Teichmann A, HNC Agra, LS Nunes, MP Rocha, JDP Renner, LG Possuelo, M Carneiro, ARieger, LB Benitez and AR Valim, 2014. Antibiotic resistance and detection of the sul2 gene in urinary isolates of Escherichia coli in patients from Brazil. J. Infect. Dev. Ctries., 8:39-43.

Trobos M, Christensen H, Sunde M, Nordentoft S, Agers $\varnothing$ Y, Simonsen GS, AM Hammerum and JE Olsen, 2009. Characterization of sulphonamide-resistant Escherichia coli using comparison of sul2 gene sequences and multilocus sequence typing. Microbiology, 155:831-836.

Vedantam G, GG Guay, NE Austria, SZ Doktor and BP Nichols, 1998.Characterization of mutations contributing to sulfathiazole resistance in Escherichia coli. Antimicrob. Agents. Chemother. 42:88-93.

Wu S, A Dalsgaard, AM Hammerum, LJ Porsbo and LB Jensen, 2010. Prevalence and characterization of plasmids carrying sulfonamide resistance genes among Escherichia coli from pigs, pig carcasses and human. Acta. Vet. Scand., 52:1-7. 